\title{
Angiography-based coronary flow reserve: The feasibility of automatic computation by artificial intelligence
}

\author{
Qiuyang Zhao ${ }^{1,2}$, Chunming $\mathrm{Li}^{1,2}$, Miao $\mathrm{Chu}^{1,2}$, Juan Luis Gutiérrez-Chico ${ }^{3}$, Shengxian $\mathrm{Tu}^{1,2}$ \\ ${ }^{1}$ Biomedical Instrument Institute, School of Biomedical Engineering, \\ Shanghai Jiao Tong University, Shanghai, China \\ ${ }^{2}$ Shanghai Med-X Engineering Research Center, Shanghai Jiao Tong University, Shanghai, China \\ ${ }^{3}$ Cardiology Department, Campo de Gibraltar Health Trust, Algeciras, Spain
}

\begin{abstract}
Background: Coronary flow reserve (CFR) has prognostic value in patients with coronary artery disease. However, its measurement is complex, and automatic methods for CFR computation are scarcely available. We developed an automatic method for CFR computation based on coronary angiography and assessed its feasibility.

Methods: Coronary angiographies from the Corelab database were annotated by experienced analysts. A convolutional neural network (CNN) model was trained for automatic segmentation of the main coronary arteries during contrast injection. The segmentation performance was evaluated using 5-fold cross-validation. Subsequently, the CNN model was implemented into a prototype software package for automatic computation of the CFR $\left(C F R_{\text {auto }}\right)$ and applied on a different sample of patients with angiographies performed both at rest and during maximal hyperemia, to assess the feasibility of $C F R_{\text {auto }}$ and its agreement with the manual computational method based on frame count (CFR $\left.R_{\text {manual }}\right)$.

Results: Altogether, 137,126 images of 5913 angiographic runs from 2407 patients were used to develop and evaluate the CNN model. Good segmentation performance was observed. CFR auto was successfully computed in 136 out of 149 vessels (91.3\%). The average analysis time to derive CFR auto was $18.1 \pm 10.3 \mathrm{~s}$ per vessel. Moderate correlation $(r=0.51, p<0.001)$ was observed between $C F R_{\text {auto }}$ and $C F R_{\text {manual }}$ with a mean difference of $0.12 \pm 0.53$.

Conclusions: Automatic computation of the CFR based on coronary angiography is feasible. This method might facilitate wider adoption of coronary physiology in the catheterization laboratory to assess microcirculatory function. (Cardiol J)
\end{abstract}

Key words: artificial intelligence, convolutional network, coronary flow reserve, X-ray angiography, coronary heart disease

\section{Introduction}

Myocardial ischemia can be due to epicardial or microvascular disease, which are the two main leading pathophysiological mechanisms. Fractional flow reserve (FFR) has consistently proven to be the most stable and accurate parameter to assess the hemodynamic severity of epicardial coronary stenosis. Guidance of percutaneous coronary interventions (PCI) by FFR results in significant improvement of outcomes in different clinical scenarios [1-3].

Address for correspondence: Shengxian Tu, PhD, FACC, FESC, Med-X Research Institute, Shanghai Jiao Tong University, No. 1954, Huashan Road, Xuhui District, Shanghai, 200030, China, tel: +86 21 62932631, fax: +86 21 62932156, e-mail: sxtu@sjtu.edu.cn

Received: 20.03.2021 Accepted: 7.05.2021 Early publication date: 2.08.2021

This article is available in open access under Creative Common Attribution-Non-Commercial-No Derivatives 4.0 International (CC BY-NC-ND 4.0) license, allowing to download articles and share them with others as long as they credit the authors and the publisher, but without permission to change them in any way or use them commercially. 
Conversely, coronary microvascular dysfunction has been classically relegated to a secondary role due to different factors, among them the complexity of its assessment. Coronary flow reserve (CFR), defined as hyperemia-to-rest flow ratio, depends on both epicardial and microvascular vessels, thus being one of the parameters used to estimate microvascular dysfunction. CFR can be non-invasively assessed by positron emission tomography [4], or invasively by means of Doppler wire [5] or thermodilution [6,7]. However, all these methods are scarcely available in most cardiology departments, being restricted to few expert centers. Nonetheless, the evidence on the prognostic impact and clinical relevance of microvascular dysfunction is currently increasing $[8,9]$ and subsequently, the need to increase the availability of a method to assess coronary microcirculatory function. Discordance between FFR and CFR assessment occurs in up to $32 \%$ of cases [10], because they offer clinically relevant complementary information. Patients without epicardial disease (normal FFR) but microvascular dysfunction (low CFR) have a significantly worse prognosis than those with both normal FFR and CFR [8]. Furthermore, a recent study has challenged the paradigm of revascularization in lesions with low FFR but preserved CFR [9]. This evidence is, however, hardly pervading clinical practice, due to the limited availability of current methods to assess CFR [11].

Image-based computational methods of physiology have substantially contributed to reducing costs and to expanding physiology guidance in PCI. FFR can be accurately estimated by different computational methods based on coronary computed tomography angiography [12], coronary angiography [13, 14], optical coherence tomography [15-17], or intravascular ultrasound [18]. Nonetheless, the feasibility of computational methods for the assessment of microcirculatory function has been limited, although some pioneer approaches have recently been proposed, with varying success $[19,20]$. In the current study, we aimed to propose a novel automatic computational approach to estimate CFR based on coronary angiography, dubbed $\mathrm{CFR}_{\text {auto, }}$, and evaluated its potential to improve the availability of microvascular assessment for clinical decision-making in a cost-effective manner.

\section{Methods}

\section{Study sample}

A search of the database of the Corelab (CardHemo, Med-X Research Institute, Shanghai Jiao
Tong University, Shanghai, China) was performed, looking for patients with coronary angiography performed at rest or/and under maximal hyperemia. The exclusion criteria were as follows: 1) Patients with chronic total occlusion; 2) Patients with prior coronary bypass grafting of the interrogated vessels; 3) Angiographic images with significant overlap or foreshortening. Data of all patients with coronary angiography performed only at rest were used for the development and validation of the convolutional neural network (CNN) model. The remaining patients with coronary angiography performed both at rest and under maximal hyperemia were used for independent validation of the $\mathrm{CFR}_{\text {auto }}$. Figure 1 shows the flow diagram of the study, explaining the use of the different datasets for $\mathrm{CNN}$ model development and $\mathrm{CFR}_{\text {auto }}$ validation. The study protocol was approved by the institutional review board, and all patients had previously provided informed consent for enrolment into the institutional database for potential future investigations.

\section{Vessel segmentation in the CNN}

Data annotation. Lumen contours of the three main epicardial coronary arteries with coronary stenosis, namely the left anterior descending (LAD), left circumflex (LCx), and right coronary artery (RCA), in all patients were semi-automatically annotated by experienced analysts, trained in quantitative coronary angiography (QCA) and regularly audited at the core laboratory.

Development of the $\mathrm{CNN}$ model. An adapted CNN model in U-shape [21] was applied for segmentation of the main epicardial coronary arteries. The model consisted of a contracting path for high-level feature extraction and an expansion path to produce a full-resolution segmentation image. Details of the architecture of the original U-Net have been described previously [21]. The U-Net was modified to optimize the segmentation of the vessel: two additional down-sampling layers were implemented on the U-Net structure to enlarge the receptive field and thus avoid discontinuity of detected centerlines. Moreover, the number of feature maps per layer was reduced to accelerate the computation speed (Fig. 2).

For the $\mathrm{CNN}$ training process, a combination of dice and focal losses was used as the loss function [22, 23]. Dice loss is widely used for image segmentation, with excellent results, while focal loss can be useful in cases of smaller vessel area with respect to the image size. The Adam optimization algorithm [24] was used to facilitate CNN 


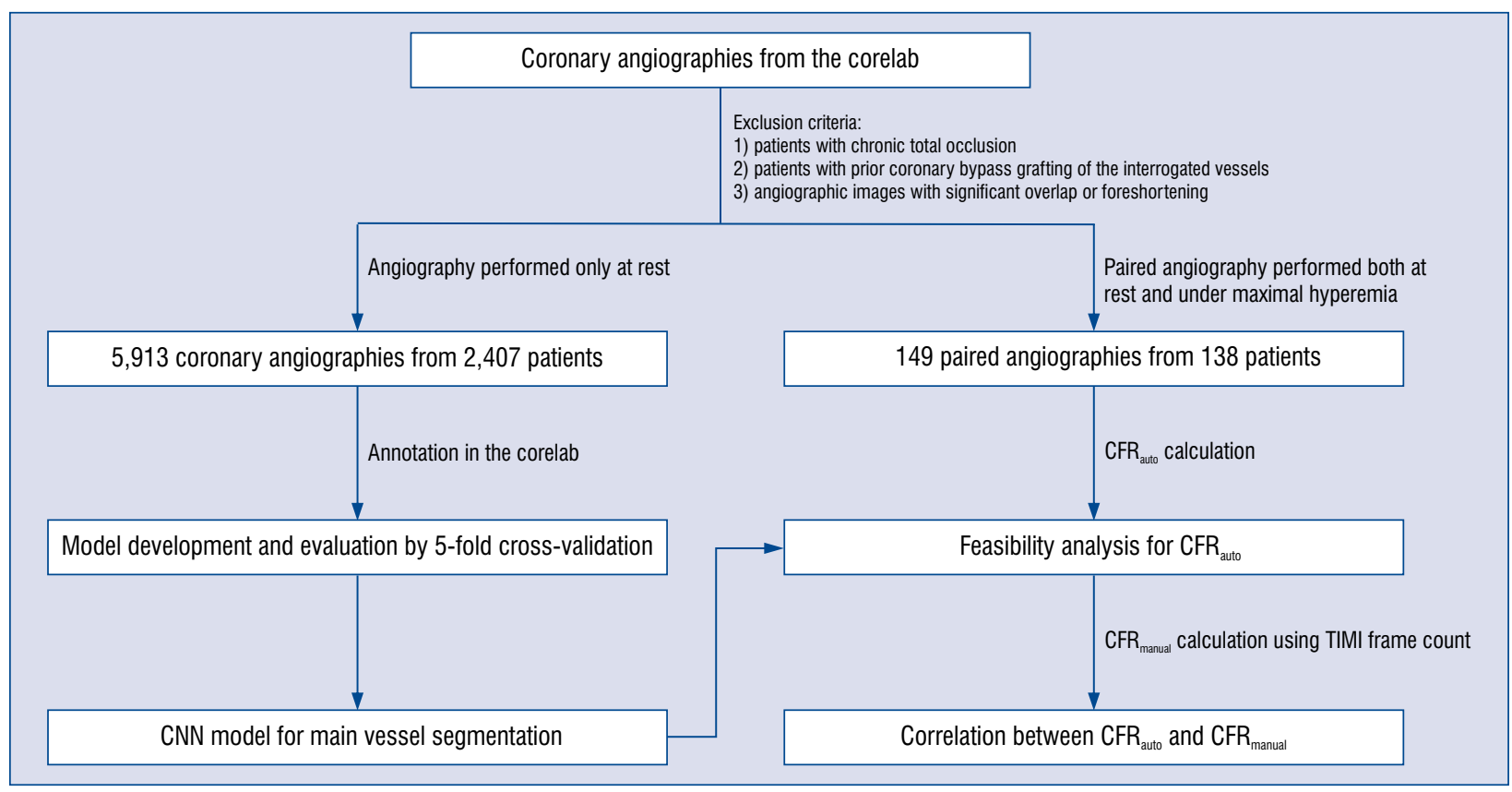

Figure 1. Flow diagram of the study; CFR - coronary flow reserve; CNN — convolutional neural network; TIMI Thombolysis in Myocardial Infarction.

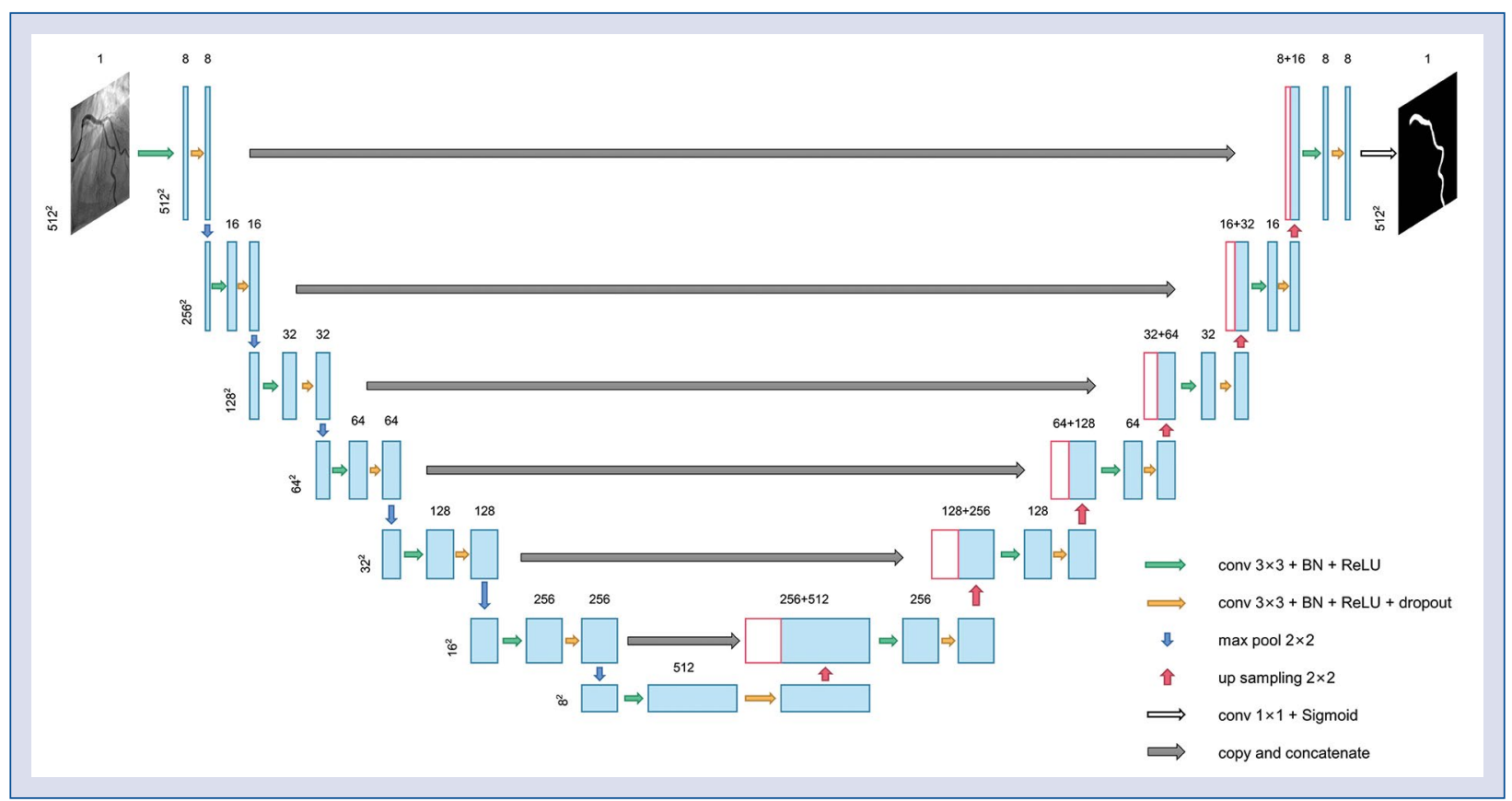

Figure 2. The structure of the proposed convolutional neural network for vessel segmentation.

convergence. The whole training process has 70 epochs in total, with a learning rate of $2 \times 10^{-4}$ at the first 30 epochs. At the $31^{\text {st }}$ and $61^{\text {st }}$ epochs, the learning rate decreased to 0.4 times that of the previous learning rate. This setting facilitates $\mathrm{CNN}$ convergence while preventing overfitting.
The CNN model for LAD, LCx, and RCA segmentation was separately trained using the corresponding datasets. Model performance was evaluated by 5 -fold cross-validation using evaluation metrics of dice similarity index, precision, recall, and $\mathrm{F} 1$ score. 


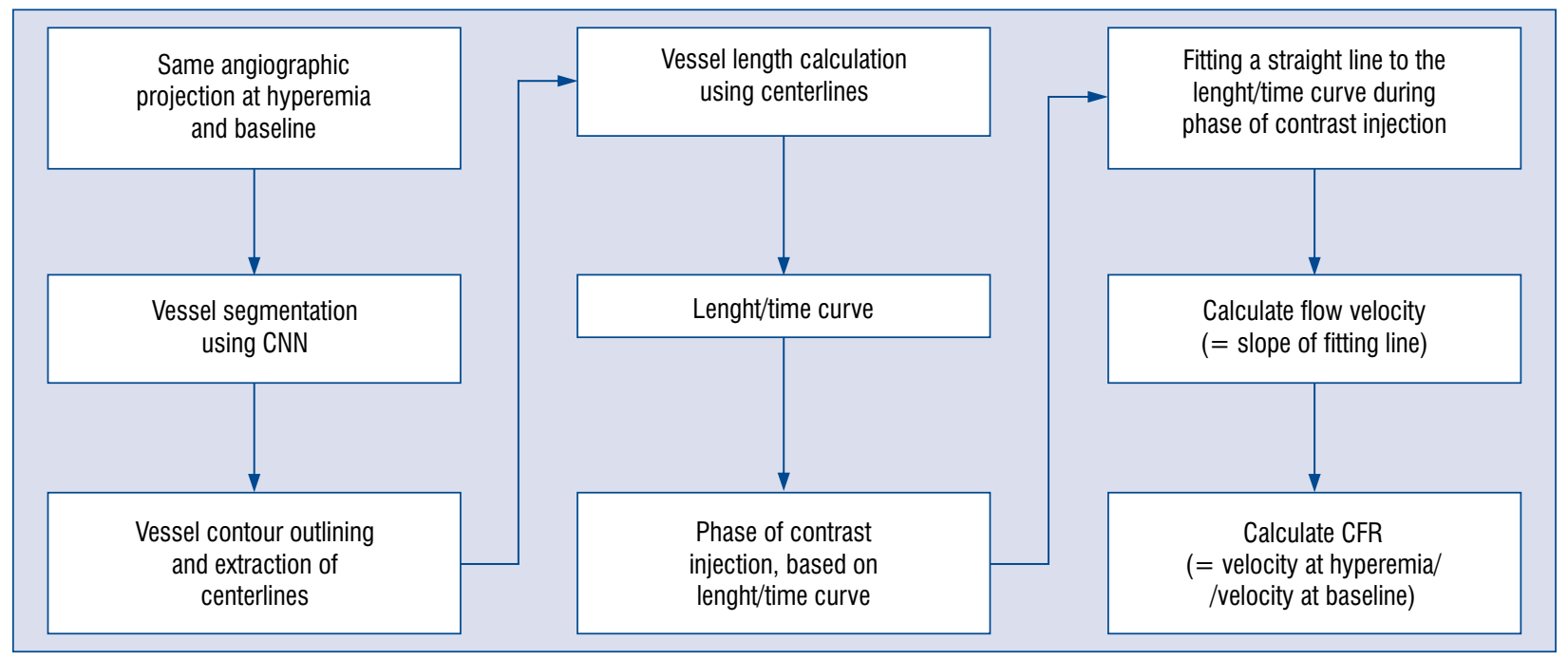

Figure 3. Workflow diagram describing the entire process of the proposed method; CFR - coronary flow reserve; $\mathrm{CNN}$ - convolutional neural network.

\section{Automatic CFR computation}

The developed CNN model was integrated into a prototype software package (FlowPlus; Pulse Medical Imaging Technology, Shanghai, China) for automatic CFR calculation. Two loops of the same angiographic projection, at hyperemia and at baseline, should be uploaded into the software in the DICOM format. The trained CNN model automatically segmented the vessel and subsequently delineated the vessel contour and centerlines. The length of the vessel was calculated for each frame according to the length of the centerline. Considering the frame rate, the curve of vessel length variation over time (length/time curve) could be easily derived. The phase of contrast injection was then automatically calculated as the period of the curve in which the length progressively increases. The flow velocity could then be easily calculated by fitting a straight line to the length/time curve during the phase of contrast injection, using the least-square method. The slope of this fitting line defined the rate of length change over time, and hence the flow velocity [25]. At this point, CFR could be derived as the quotient between hyperemic and rest flow velocities (Fig. 3).

\section{Validation of the automatic CFR computation}

The automatically computed CFR ( $\left.\mathrm{CFR}_{\text {auto }}\right)$ was validated on a different sample of patients, other than the one used for the development of the $\mathrm{CNN}$ model, considering manually calculated CFR $\left(\mathrm{CFR}_{\text {manual }}\right)$ as reference. A manual Thrombolysis in Myocardial Infarction (TIMI) frame count [26] was performed by experienced QCA analysts on the same angiography loops as the automatic count in all patients. TIMI frame count method needs to count the frames the contrast take from to point contrast just enter the vessel to the point of maximal filling of the vessel, then use the frame rate to obtain the filling time. Finally, the manually measured vessel length was used to calculate the flow velocity. Coronary flow velocities at hyperemia and baseline were then calculated, and subsequently the $\mathrm{CFR}_{\text {manual }}$ was derived, following the same rationale as previously described. The main difference between $\mathrm{CFR}_{\text {auto }}$ and $\mathrm{CFR}_{\text {manual }}$ is the way the flow velocities are derived; in $\mathrm{CFR}_{\text {auto }} \mathrm{CNN}$ was used to automatically obtain flow velocities, while in $\mathrm{CFR}_{\text {manual }}$ the flow velocity was manually calculated by counting frames. The agreement and correlation between $\mathrm{CFR}_{\text {auto }}$ and $\mathrm{CFR}_{\text {manual }}$ were then evaluated.

\section{Statistical analysis}

Continuous variables were presented as mean \pm standard deviation or median (Q1-Q3) if a Gaussian distribution could not be assumed. The correlation between $\mathrm{CFR}_{\text {auto }}$ and $\mathrm{CFR}_{\text {manual }}$ was evaluated using Pearson's correlation test and linear regression analysis, taking $\mathrm{CFR}_{\text {manual }}$ as the standard reference. The proportional bias (slope from 1) and constant bias (the deviation of the intercept from 0 ) were evaluated in the linear regression. The agreement between $\mathrm{CFR}_{\text {auto }}$ and $\mathrm{CFR}_{\text {manual }}$ as continuous variables was assessed using Bland-Altman analysis and the intraclass correlation coefficient for the absolute value (ICCa). A two-sided p-value $\leq 0.05$ was considered to indicate a statistically significant 
difference. Statistical analysis was performed using the MedCalc 18.2.1 (MedCalc Software Ltd, Acacialaan 22, 8400 Ostend, Belgium) software package.

\section{Results}

\section{Baseline demographic data}

Altogether, 137,126 images of 5913 coronary angiographic runs from 2407 patients were used for the development of the CNN model, comprising 2543 LAD, 1538 LCx, and 1832 RCA runs. The independent sample was used to assess the feasibility of the CFR auto and its agreement with the $\mathrm{CFR}_{\text {manual, }}$, which consisted of 149 paired angiographies from 138 patients. Clinical and lesion characteristics of the independent sample are presented in Tables 1 and 2 , respectively.

\section{Segmentation performance of the CNN}

The segmentation performances of the proposed CNN model were good in all epicardial coronary arteries by 5 -fold cross-validation, with mean dice coefficients values of $0.780 \pm 0.007,0.722 \pm$ \pm 0.005 , and $0.758 \pm 0.003$ for LAD, LCx, and RCA, respectively (Table 3 ). Figure 4 shows paradigmatic examples of the segmentation results for different vessels during contrast injection. Figure 5 shows some unsuccessful $\mathrm{CFR}_{\text {auto }}$ computations.

\section{Feasibility of automatic CFR analysis}

$\mathrm{CFR}_{\text {auto }}$ computation was successful in $\mathbf{1 3 6}$ out of 149 vessels (feasibility $91.3 \%$ ). Unsuccessful $\mathrm{CFR}_{\text {auto }}$ computations were due to poor visualization of contrast dye flowing $(n=7)$, missegmentation of the catheter $(\mathrm{n}=4)$, interposition of other anatomic structures $(\mathrm{n}=1)$, and unusual angiographic view $(\mathrm{n}=1)$.

\section{Correlation and agreement analysis}

The average value of $\mathrm{CFR}_{\text {auto }}$ was $1.49 \pm$ \pm 0.54 . Moderate correlation $(\mathrm{r}=0.51, \mathrm{p}<0.001)$ was observed between $\mathrm{CFR}_{\text {auto }}$ and $\mathrm{CFR}_{\text {manual }}$, with a slope 0.511 and an intercept 0.857 in the linear re-

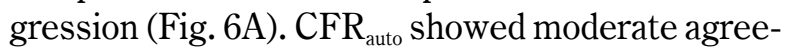
ment with $\mathrm{CFR}_{\text {manual }}$ (mean difference $=0.12 \pm$
Table 1. Baseline clinical characteristics ( $n=138$ ).

\begin{tabular}{lc}
\hline Age [years] & $63.7 \pm 9.2$ \\
Male & $108(78.2)$ \\
Body mass index $\left[\mathrm{kg} / \mathrm{m}^{2}\right]$ & $26.7(24.2-29.5)^{*}$ \\
Hypertension & $79(57.2 \%)$ \\
Diabetes mellitus & $37(29.1 \%)^{*}$ \\
Cardiovascular history: & \\
Prior myocardial infarction & $38(27.5 \%)$ \\
Prior PCl & $50(36.2 \%)$ \\
Prior CABG & $5(3.6 \%)$ \\
\hline
\end{tabular}

Values are mean \pm standard deviation, number $(\%)$ or median (interquartile range). *Body mass index missing in 4 patients, diabetes mellitus missing in 11 patients; CABG - coronary artery bypass surgery; $\mathrm{PCl}$ - percutaneous coronary intervention

Table 2. Baseline lesion characteristics $(n=149)$.

\begin{tabular}{ll}
\hline Index artery: & \\
Left anterior descending artery & $85(57.0 \%)$ \\
Left circumflex artery & $34(22.8 \%)$ \\
Right coronary artery & $30(20.1 \%)$ \\
Percent diameter stenosis [\%] & $46.3 \pm 8.2$ \\
Minimum lumen diameter [mm] & $1.52 \pm 0.36$ \\
Reference vessel diameter [mm] & $2.82 \pm 0.46$ \\
\hline
\end{tabular}

Values are number (\%) and mean \pm standard deviation.

$\pm 0.53, \mathrm{p}<0.001, \mathrm{ICCa}=0.50 ; 95 \%$ confidence interval [CI] 0.36-0.62) (Fig. 6B). Inter- and intraobserver variability in $\mathrm{CFR}_{\text {manual }}$ calculation were $0.09 \pm 0.74$ and $0.03 \pm 0.42$, with ICCa values of 0.62 (95\% CI $0.41-0.76)$ and 0.71 (95\% CI $0.54-0.83)$, respectively.

Analysis time of automatic CFR computation The average analysis time for computation of CFR per vessel was $18.1 \pm 10.3 \mathrm{~s}$ on an off-theshelf workstation equipped with a 6-core Intel i7$8750 \mathrm{H}$ processor (Intel Corporation, Santa Clara, CA, USA; 2.2 GHZ), NVIDIA GeForce GTX 1050Ti graphics card (NVIDIA, Santa Clara, CA, USA), and 16 GB of RAM.

Table 3. Segmentation performance of the proposed model on left anterior descending (LAD), left circumflex (LCX), and right coronary artery (RCA) vessels using 5 -fold cross-validation.

\begin{tabular}{lcccc}
\hline & Dice & Precision & Recall & F1 \\
\hline LAD & $0.780 \pm 0.007$ & $0.763 \pm 0.004$ & $0.919 \pm 0.004$ & $0.834 \pm 0.002$ \\
LCx & $0.722 \pm 0.005$ & $0.748 \pm 0.004$ & $0.849 \pm 0.005$ & $0.796 \pm 0.003$ \\
RCA & $0.758 \pm 0.003$ & $0.777 \pm 0.006$ & $0.893 \pm 0.004$ & $0.831 \pm 0.002$ \\
\hline
\end{tabular}




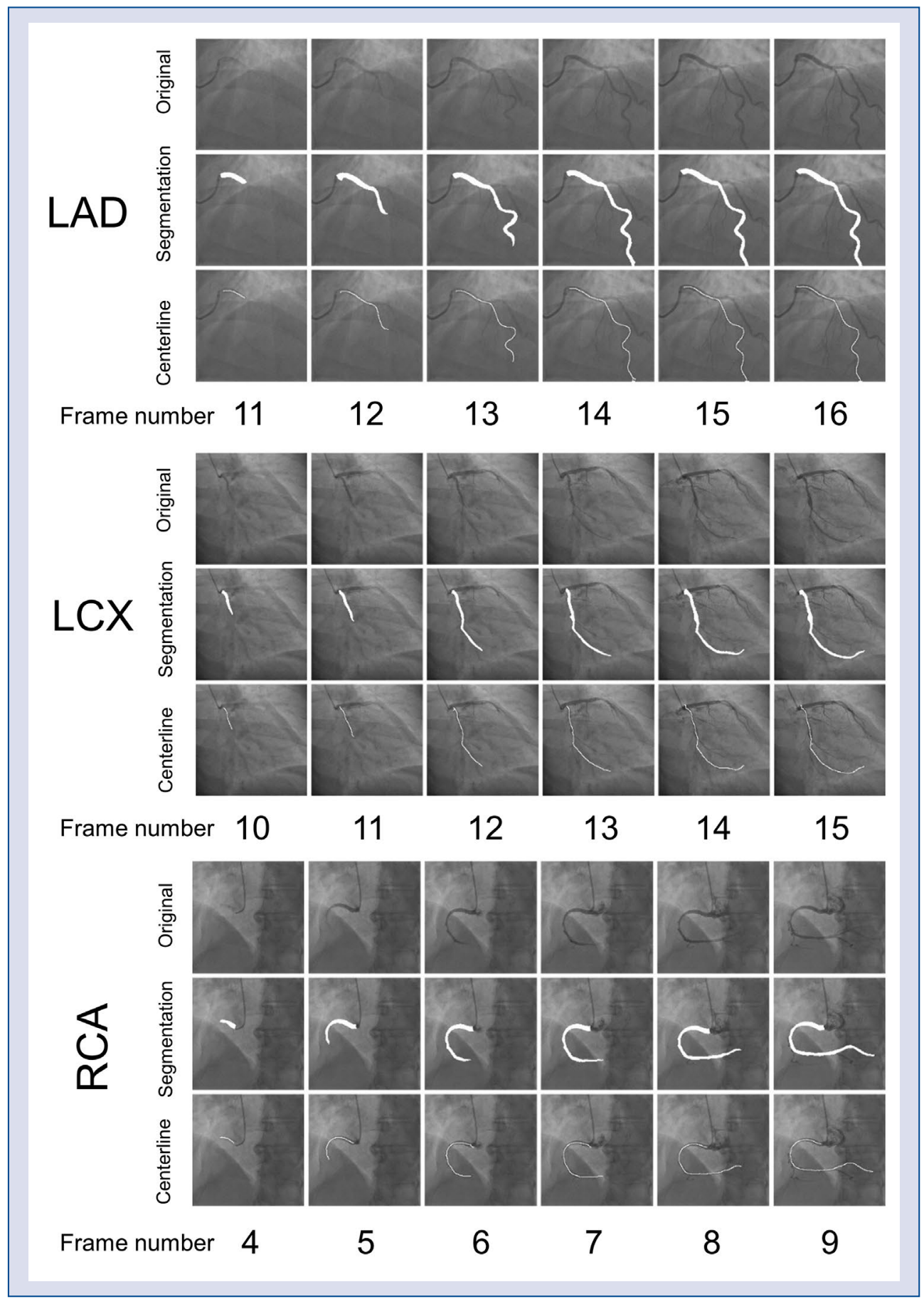

Figure 4. Segmentation results of left anterior descending (LAD), left circumflex (LCx), and right coronary artery (RCA). The first row of each group is the original image, the second row is the segmentation result, and the third row is the extracted vessel centerline. The white area is the segmentation mask and centerline mask.

\section{Discussion}

To the best of our knowledge, this is the first study validating a novel method based on artificial intelligence for automatic CFR computation from coronary angiography. The key findings of the present study are as follows: 1) The CNN model showed good performance in segmentation of the main coronary arteries from angiographic loops, 2) Automatic CFR calculation from coronary angiography is feasible in $91.3 \%$ of cases; 3 ) Automatic CFR computation showed moderate agreement 


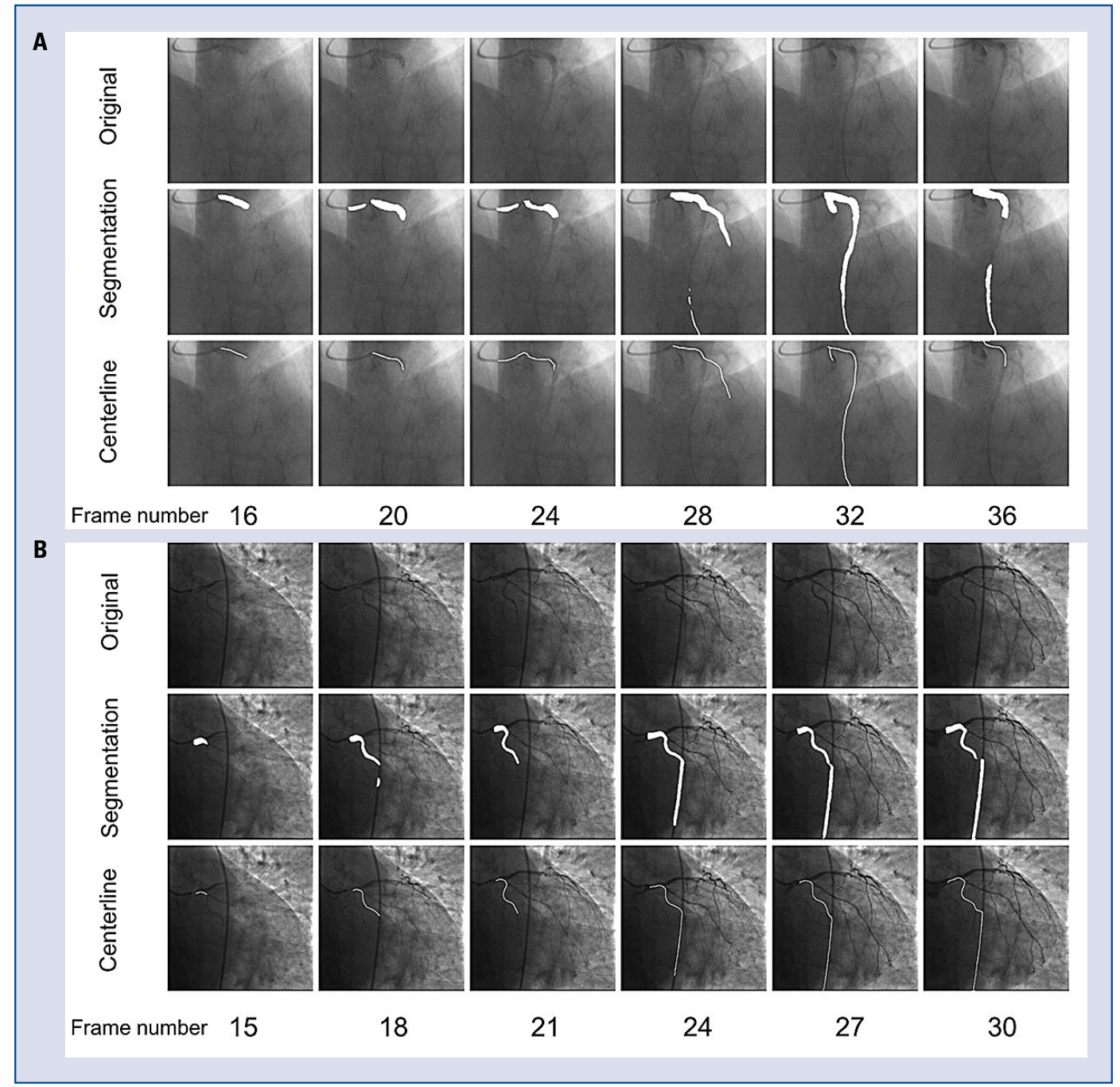

Figure 5. Paradigmatic cases of unsuccessful automatic coronary flow reserve computations. The first row of each group is the original image, the second row is the segmentation result, and the third row is the extracted vessel centerline. Common mechanisms for failure are poor visualization of contrast dye (A), and mis-segmentation of the catheter (B) or of other anatomic structures.

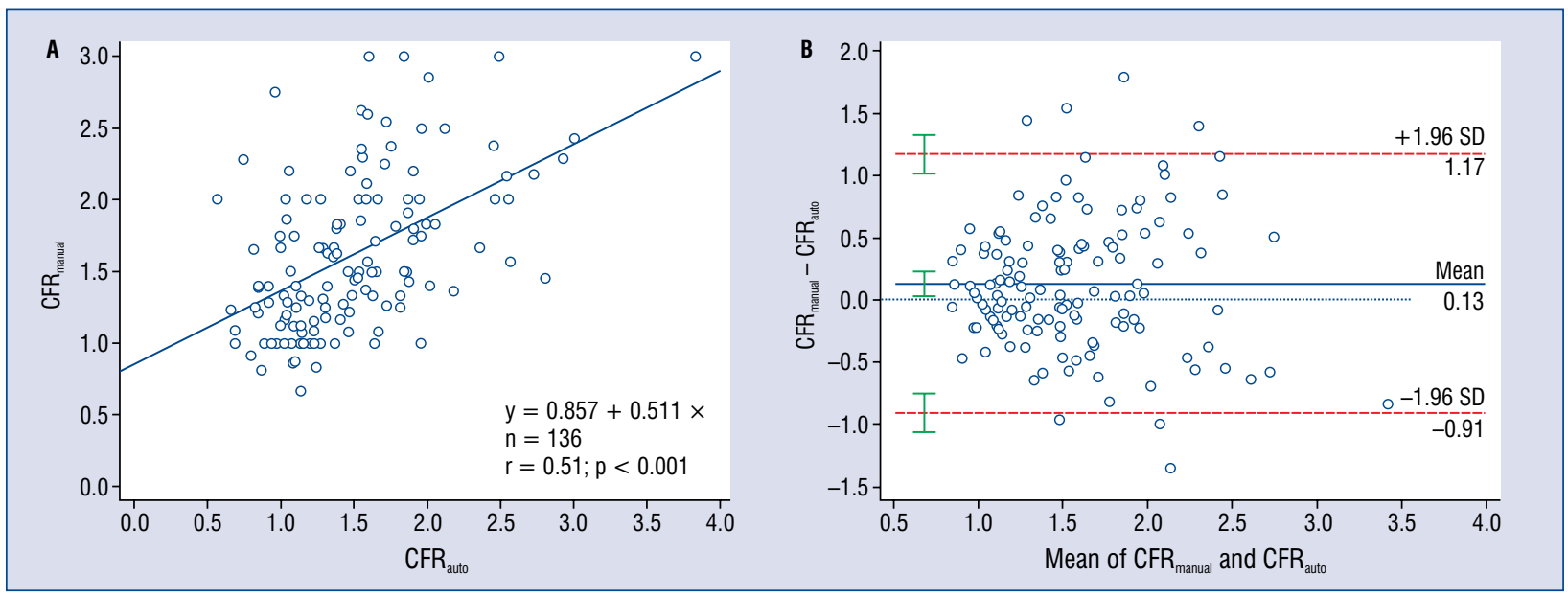

Figure 6. Correlation and agreement between automatic coronary flow reserve $\left(\mathrm{CFR}_{\text {auto }}\right)$ and frame-counting $\mathrm{CFR}$ (CFR manual $_{\text {). }}$. 
with conventional manual CFR calculation based on TIMI frame count.

Physiology-guidance has consistently proved to result in better clinical outcomes than classical anatomic-guidance for the treatment of epicardial coronary disease in different clinical scenarios [1-3, 27, 28]. Nonetheless, patients with microvascular disease have historically been neglected because of the scarce availability of methods to assess microcirculatory function. The access to positron emission tomography, Doppler wire, or thermodilution remains limited in most cardiology departments worldwide, so patients with microvascular dysfunction are often denied a diagnosis. The cases are instead considered non-cardiologic and are referred for endless gastroenterological or psychiatric studies, thus resulting in considerable frustration and depression, together with unnecessary costs for the health systems. The evidence on the prognostic relevance of microcirculatory dysfunction is currently compelling [8], and therefore the need to generalize its evaluation is as unmet. A cost-effective angiography-based method to estimate CFR could exponentially increase the availability of microvascular assessment, without additional wiring or prolongation of the procedure. Some groups have proposed angiography-based computational methods to assess the index of microvascular resistance (IMR), a parameter to specifically appraise the microcirculatory function, finding acceptable agreement with thermodilution [19]. Our study follows a similar approach, although it focuses on CFR and developing a model of artificial intelligence to simplify the calculation in the cathlab. The high feasibility of the proposed method (91.3\%) suggests a broad practical applicability. In this first step, the CNN model focused on CFR calculation, a parameter depending on both epicardial and microvascular functions, although other parameters to assess microvascular dysfunction, like IMR, could similarly be derived in future studies following a similar rationale.

The applications of artificial intelligence in the field of coronary artery segmentation from angiography are expanding. Different $\mathrm{CNN}$ architectures have been proposed to segment the entire coronary tree [29, 30] or the main vessel [31]. Of note, our segmentation task is unique because all frames of the angiographic run covering the entire contrast injection were segmented. At early phases of the contrast injection, the main vessels appear short in length and the definition of the borders is poorer than at phases of complete filling, thus increasing the segmentation difficulty and potentially affect- ing the evaluation of the overall segmentation performance.

For all procedures involving artificial intelligence, the first mandatory step is the validation vs. the same procedure manually performed by expert human operators. Thus, the current study validated an automatic method to calculate CFR, based on artificial intelligence, vs. the same manual computational method. This was not a validation of the computational method vs. an invasive standard, as previous studies have done [19,20]. This kind of validation will be pursued in future studies once the CNN model has been fine-tuned, and it may provide interesting complementary information. It might help to understand the moderate agreement between manual and automatic methods for CFR calculation, notwithstanding the excellent performance of the $\mathrm{CNN}$ model for the segmentation of the vessels. The correlation between $\mathrm{CFR}_{\text {auto }}$ and $\mathrm{CFR}_{\text {manual }}$ is moderate. However, it is important to note that the manual computational method based on frame count is not the clinical standard for CFR measurement. The reproducibility of $\mathrm{CFR}_{\text {manual }}$ is only moderate, as indicated by the inter- and intraobserver ICCa of 0.62 (95\% CI 0.41-0.76) and 0.71 (95\% CI 0.54-0.83), respectively. Moreover, the values found in linear regression with a slope and an intercept that considerably deviated from 1 and 0 , respectively, do not permit us to rule out proportional or constant bias. This may be because the manual operators tend to count frames outside the steady perfusion period, especially at hyperemia and at high flow velocities, resulting in higher flow velocities and CFR values than the automatic method, especially at the high extreme of the scale. However, the CNN model might be more consistent, accurate, and reproducible than the corresponding manual method, as in other CNN models [32]. Therefore, we consider that the variability of $\mathrm{CFR}_{\text {manual }}$ played a major role in the moderate correlation between $\mathrm{CFR}_{\text {auto }}$ and $\mathrm{CFR}_{\text {manual }}$. The validation of the method vs. an invasive standard might show that the CNN models outperform the manual calculation or otherwise unravel details of the workflow that might eventually deserve further attention.

\section{Limitations of the study}

The CNN model focused exclusively on the calculation of CFR, a parameter that depends on both epicardial and microvascular function. Other parameters, like IMR, are more specific to assessing microvascular dysfunction, and they could be similarly derived in future studies, following a similar rationale. As in other computational meth- 
ods of physiology, this approach loses accuracy in some anatomical scenarios, such as chronic total occlusions or bypass grafts, and might have limited feasibility in cases of extreme foreshortening or vessel overlap, which were excluded from the current study [13-15, 17, 33].

\section{Conclusions}

Automatic CFR computation using coronary angiography was feasible and showed a moderate agreement with the manual computational method based on frame count. Image-derived CFR calculation may facilitate wider adoption of coronary physiology and the assessment of microvascular function in routine clinical practice.

\section{Funding}

This work was supported by the Natural Science Foundation of China (grant numbers 682020108015 and 81871460) and by the Science and Technology Commission of Shanghai Municipality (grant number 19DZ1930600).

Conflict of interest: Shengxian Tu received a research grant from Pulse Medical Imaging Technology.

\section{References}

1. Bech GJ, De Bruyne B, Pijls NH, et al. Fractional flow reserve to determine the appropriateness of angioplasty in moderate coronary stenosis: a randomized trial. Circulation. 2001; 103(24): 2928-2934, doi: 10.1161/01.cir.103.24.2928, indexed in Pubmed: 11413082.

2. De Bruyne B, Pijls NHJ, Kalesan B, et al. FAME 2 Trial Investigators. Fractional flow reserve-guided $\mathrm{PCI}$ versus medical therapy in stable coronary disease. N Engl J Med. 2012; 367(11): 991-1001, doi: 10.1056/NEJMoa1205361, indexed in Pubmed: 22924638

3. Tonino P, Bruyne BDe, Pijls N, et al. Fractional flow reserve versus angiography for guiding percutaneous coronary intervention. N Engl J Med. 2009; 360(3): 213-224, doi: 10.1056/ nejmoa0807611.

4. Driessen RS, Raijmakers PG, Stuijfzand WJ, et al. Myocardial perfusion imaging with PET. Int J Cardiovasc Imaging. 2017; 33(7): 1021-1031, doi: 10.1007/s10554-017-1084-4, indexed in Pubmed: 28188475.

5. Doucette JW, Corl PD, Payne HM, et al. Validation of a Doppler guide wire for intravascular measurement of coronary artery flow velocity. Circulation. 1992; 85(5): 1899-1911, doi: 10.1161/01.cir.85.5.1899, indexed in Pubmed: 1572046.

6. Barbato E, Aarnoudse W, Aengevaeren WR, et al. Week 25 study group. Validation of coronary flow reserve measurements by thermodilution in clinical practice. Eur Heart J. 2004; 25(3): 219-223, doi: 10.1016/j.ehj.2003.11.009, indexed in Pubmed: 14972422 .

7. Pijls NHJ, De Bruyne B, Smith L, et al. Coronary thermodilution to assess flow reserve: validation in humans. Circulation. 2002;
105(21): 2482-2486, doi: 10.1161/01.cir.0000017199.09457.3d, indexed in Pubmed: 12034653.

8. Lee JM, Jung JH, Hwang D, et al. Coronary flow reserve and microcirculatory resistance in patients with intermediate coronary stenosis. J Am Coll Cardiol. 2016; 67(10): 1158-1169, doi: 10.1016/j.jacc.2015.12.053, indexed in Pubmed: 26965536.

9. Stegehuis VE, Wijntjens GWM, van de Hoef TP, et al. Distal Evaluation of Functional performance with Intravascular sensors to assess the Narrowing Effect-combined pressure and Doppler FLOW velocity measurements (DEFINE-FLOW) trial: Rationale and trial design. Am Heart J. 2020; 222: 139-146, doi: 10.1016/j. ahj.2019.08.018, indexed in Pubmed: 32062172.

10. Ahn SG, Suh J, Hung OY, et al. Discordance between fractional flow reserve and coronary flow reserve: insights from intracoronary imaging and physiological assessment. JACC Cardiovasc Interv. 2017; 10(10): 999-1007, doi: 10.1016/j.jcin.2017.03.006, indexed in Pubmed: 28521932.

11. Lee HS, Lee JM, Nam CW, et al. Consensus document for invasive coronary physiologic assessment in Asia-Pacific countries. Cardiol J. 2019; 26(3): 215-225, doi: 10.5603/CJ.a2019.0054, indexed in Pubmed: 31225632.

12. Douglas PS, Pontone G, Hlatky MA, et al. Clinical outcomes of fractional flow reserve by computed tomographic angiographyguided diagnostic strategies vs. usual care in patients with suspected coronary artery disease: the prospective longitudinal trial of FFR(CT): outcome and resource impacts study. Eur Heart J. 2015; 36(47): 3359-3367, doi: 10.1093/eurhearti/ehv444, indexed in Pubmed: 26330417.

13. Tu S, Barbato E, Köszegi Z, et al. Fractional flow reserve calculation from 3-dimensional quantitative coronary angiography and TIMI frame count: a fast computer model to quantify the functional significance of moderately obstructed coronary arteries. JACC Cardiovasc Interv. 2014; 7(7): 768-777, doi: 10.1016/j. jcin.2014.03.004, indexed in Pubmed: 25060020.

14. Tu S, Westra J, Yang J, et al. FAVOR Pilot Trial Study Group. Diagnostic Accuracy of Fast Computational Approaches to Derive Fractional Flow Reserve From Diagnostic Coronary Angiography: The International Multicenter FAVOR Pilot Study. JACC Cardiovasc Interv. 2016; 9(19): 2024-2035, doi: 10.1016/j. jcin.2016.07.013, indexed in Pubmed: 27712739.

15. Gutiérrez-Chico JL, Chen Y, Yu W, et al. Diagnostic accuracy and reproducibility of optical flow ratio for functional evaluation of coronary stenosis in a prospective series. Cardiol J. 2020; 27(4): 350-361, doi: 10.5603/CJ.a2020.0071, indexed in Pubmed: 32436590 .

16. Huang J, Emori H, Ding D, et al. Comparison of Diagnostic Performance of Intracoronary Optical Coherence Tomography-based and Angiography-based Fractional Flow Reserve for Evaluation of Coronary Stenosis. Eurointervention: Journal of Europcr in Collaboration with the Working Group on Interventional Cardiology of the European Society of Cardiology. 2020.

17. Yu W, Huang J, Jia D, et al. Diagnostic accuracy of intracoronary optical coherence tomography-derived fractional flow reserve for assessment of coronary stenosis severity. EuroIntervention. 2019; 15(2): 189-197, doi: 10.4244/EIJ-D-19-00182, indexed in Pubmed: 31147309.

18. Yu W, Tanigaki T, Ding D, et al. TCT CONNECT-202 Diagnostic Accuracy of Novel Ultrasonic Flow Ratio in Identifying Hemodynamical Significance of Coronary Stenosis. J Am Coll Cardiol. 2020; 76(17): B86, doi: 10.1016/j.jacc.2020.09.217.

19. De Maria GL, Scarsini R, Shanmuganathan M, et al. Angiography-derived index of microcirculatory resistance as a novel, 


\section{Cardiology Journal}

pressure-wire-free tool to assess coronary microcirculation in ST elevation myocardial infarction. Int J Cardiovasc Imaging. 2020; 36(8): 1395-1406, doi: 10.1007/s10554-020-01831-7, indexed in Pubmed: 32409977.

20. Tebaldi M, Biscaglia S, Di Girolamo D, et al. Angio-Based index of microcirculatory resistance for the assessment of the coronary resistance: a proof of concept study. J Interv Cardiol. 2020; 2020: 8887369, doi: 10.1155/2020/8887369, indexed in Pubmed: 33162844.

21. Ronneberger O, Fischer P, Brox T. U-Net: Convolutional Networks for Biomedical Image Segmentation. Lecture Notes in Computer Science. 2015: 234-241, doi: 10.1007/978-3-319-24574-4_28.

22. Milletari F, Navab N, Ahmadi SA. V-Net: Fully Convolutional Neural Networks for Volumetric Medical Image Segmentation. 2016 Fourth International Conference on 3D Vision (3DV). 2016, doi: $10.1109 / 3$ dv.2016.79.

23. Lin TY, Goyal P, Girshick R, et al. Focal Loss for Dense Object Detection. 2017 IEEE International Conference on Computer Vision (ICCV). 2017: 2980-2988, doi: 10.1109/iccv.2017.324.

24. Kingma DP, Ba J. Adam: A method for stochastic optimization. arXiv preprint arXiv. 2014; 14126980.

25. Zhang Y, Zhang Su, Westra J, et al. Automatic coronary blood flow computation: validation in quantitative flow ratio from coronary angiography. Int J Cardiovasc Imaging. 2019; 35(4): 587-595, doi: 10.1007/s10554-018-1506-y, indexed in Pubmed: 30535657.

26. TIMI Study Group. The Thrombolysis in Myocardial Infarction (TIMI) trial. Phase I findings. N Engl J Med. 1985; 312(14): 932-936, doi: 10.1056/NEJM198504043121437, indexed in Pubmed: 4038784 .
27. Davies J, Sen S, Dehbi HM, et al. Use of the instantaneous wavefree ratio or fractional flow reserve in PCI. N Engl J Med. 2017; 376(19): 1824-1834, doi: 10.1056/nejmoa1700445.

28. Götberg M, Christiansen EH, Gudmundsdottir IJ, et al. Instantaneous wave-free ratio versus fractional flow reserve to guide PCI. N Engl J Med. 2017; 376(19): 1813-1823, doi: 10.1056/ NEJMoa1616540, indexed in Pubmed: 28317438.

29. Nasr-Esfahani E, Karimi N, Jafari MH, et al. Segmentation of vessels in angiograms using convolutional neural networks. Biomedical Signal Processing and Control. 2018; 40: 240-251, doi: 10.1016/j.bspc.2017.09.012.

30. Fan J, Yang J, Wang Y, et al. Multichannel fully convolutional network for coronary artery segmentation in X-ray angiograms. IEEE Access. 2018; 6: 44635-44643, doi: 10.1109/access.2018.2864592.

31. Yang Su, Kweon J, Roh JH, et al. Deep learning segmentation of major vessels in X-ray coronary angiography. Sci Rep. 2019; 9(1): 16897, doi: 10.1038/s41598-019-53254-7, indexed in Pubmed: 31729445.

32. Wu P, Gutiérrez-Chico JL, Tauzin H, et al. Automatic stent reconstruction in optical coherence tomography based on a deep convolutional model. Biomed Opt Express. 2020; 11(6): 3374-3394, doi: 10.1364/BOE.390113, indexed in Pubmed: 32637261.

33. Huang J, Emori H, Ding D, et al. Diagnostic performance of intracoronary optical coherence tomography-based versus angiography-based fractional flow reserve for the evaluation of coronary lesions. EuroIntervention. 2020; 16(7): 568-576, doi: 10.4244/ EIJ-D-19-01034, indexed in Pubmed: 31951207. 